\title{
Vibration Suppression of a 3-DOF Parallel Manipulator Based on Hybrid Negative Impulses Multi-mode Input Shaping
}

\author{
Yanyan Han ${ }^{a}$, Bing $\mathrm{Li}^{\mathrm{b}^{*}}$, Yulan Wei ${ }^{\mathrm{c}}$, Shouxin Zhu ${ }^{\mathrm{d}}$, Yingjun Dai \\ School of Information and Engineering, Huzhou Teachers College, Huzhou, 313000, P.R.China \\ ayanyanh1990@sina.com, b bingli@hutc.zj.cn (corresponding author), chappyyulan@sina.com, \\ dzsx@hutc.zj.cn, ${ }^{\mathrm{e}}$ 08082504@163.com
}

Key words: Residual vibration, Negative impulses, Multiple-mode, Hybrid input shaping

\begin{abstract}
The classic multi-mode negative impulses input shapers can suppress the residual vibration of the multi-mode system effectively. But when these several frequencies bandwidths and amplitudes of vibration modes are greatly different, the time delay and the suppression performances of input shapers are decreased. However, the hybrid multi-mode negative impulses input shapers can overcome the disadvantage. The hybrid double-mode negative impulses input shapers of a 3-DOF parallel manipulator and are constructed and compared with the classic multi-mode negative impulses input shapers. And the numerical simulations are shown out, for different frequencies bandwidths and amplitudes of vibration, and the hybrid multi-mode negative impulses input shapers can increase the total suppression performance of input shaper.
\end{abstract}

\section{Introduction}

In order to improve the speed and accuracy of the system movements, the lightweight linkages of manipulators are widely used in modern equipment and machinery and aerospace fields [1-2]. However, the lightweight linkages increase vibration and make the motion trajectory of manipulators inaccurate [3-4]. The input shapers can overcome the disadvantage. But the classic multi-mode input shapers can not effectively suppress the residual vibration of the multi-mode flexible systems with multiple natural frequencies and damping ratios which is for different frequencies bandwidths and amplitudes of vibration, whereas the total suppression performances of the input shaper are decreased. Then the hybrid multi-mode negative impulses input shapers can overcome the disadvantage.

First, the theory of hybrid multi-mode negative impulses input shapers are introduced. Second, the structure, theory and the dynamic equations of a 3-DOF parallel manipulator are expressed. Third, this paper is established the hybrid double-mode negative impulses input shapers of the 3-DOF parallel manipulator which is compared with the classic multi-mode negative impulses input shapers. Finally, the numerical simulations are carried out, for different frequencies bandwidths and amplitudes of vibration, the hybrid multi-mode negative impulses input shapers can increase the total suppression performance of input shaper.

\section{Methodologies of the Hybrid Multi-Mode Negative Impulses Input Shaper}

The Method of Multi-mode Negative Impulses Input Shaping. Input shaping is a simple and effective feedback control method that a series of input signals is convolved with a set of impulses, and then input the decomposition signals to the system to suppress the residual vibration of the flexible system [1]. And these pulse amplitude and time can be calculated by vibration frequencies and damping ratios of the system.

The Method of Single-mode Negative Impulses Input Shaper. The single-mode negative impulses input shapers eliminate the delay time of the system response of positive impulses input shapers, and increase the insensitivity of the system at the same time [5-6]. It requires that the partial sum of the 
amplitude of continuous impulses should be less than or equal to value $Q$ [7]. For example, an input shaper is composed of three negative pulses; the each pulse amplitude is $A_{1}, A_{2}, A_{3}$, the constraint equations can be written as

$$
A_{1} \leq|Q|, \quad A_{1}+A_{2} \leq|Q|, \quad A_{1}+A_{2}+A_{3} \leq|Q|
$$

The procedure of the input shaper is composed of three negative impulses shown in Fig. 1.
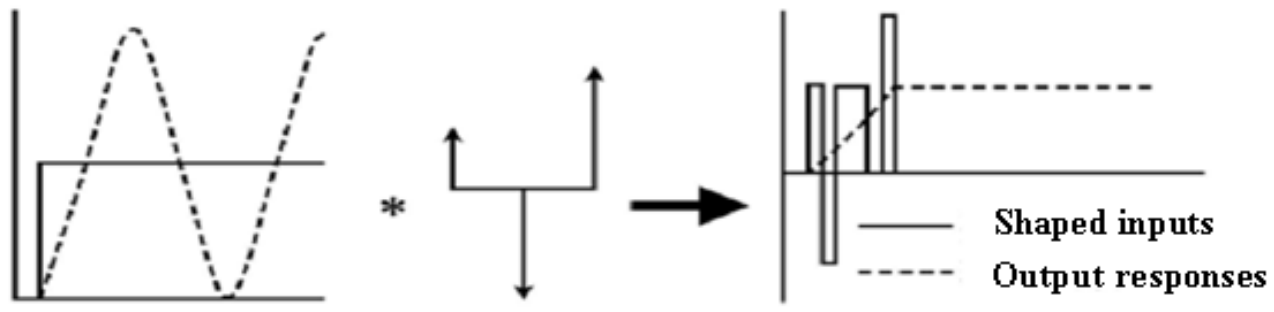

Fig. 1. Input shaping process involving negative impulses

The Theory of Classic Multi-mode Negative Impulses Input Shaper. A multiple-mode flexible system with multiple natural frequencies and damping ratios can form multiple modes which is convolved of the respective single-mode input shaper [8-9], thus constitute the different combinations of multi-mode input shaper shown in Fig. 2.

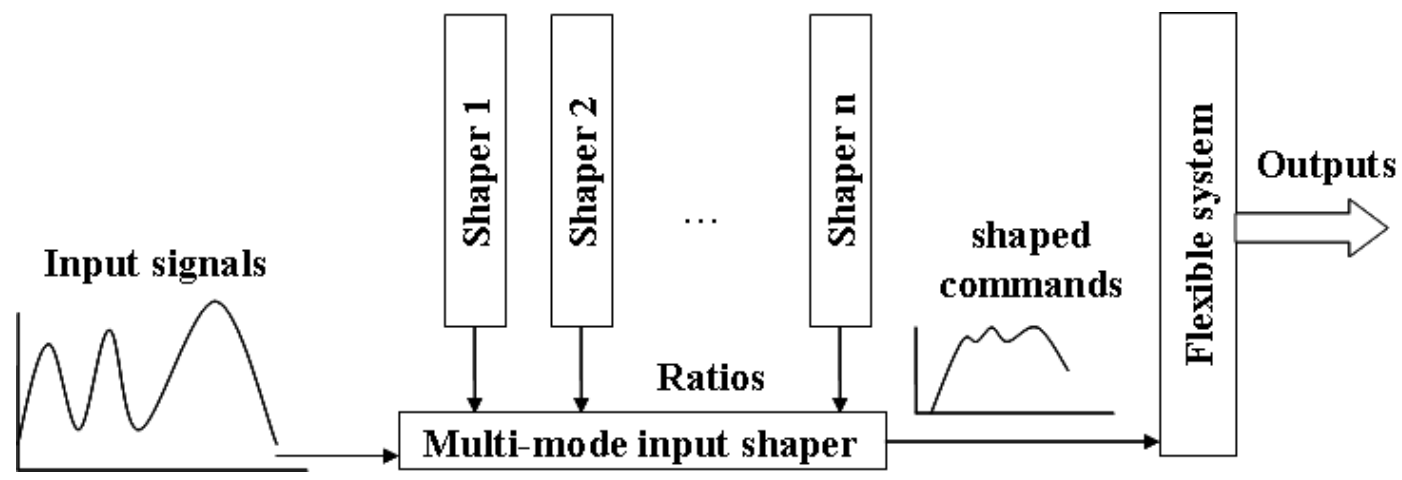

Fig. 2. Multi-mode input shaping

Based on the amplitude constraint Eq. 1 can calculated each single-mode NZV, NZVD and NEI input shapers, and then these single-mode input shapers are convolved and constitute multi-mode negative impulses input shaper.

The Hybrid Multi-mode Negative Impulses Input Shapers. When several frequencies bandwidths and amplitudes of vibration modes are greatly different, the classic multi-mode negative impulses input shapers decrease the suppression performance of flexible systems. Different systems use different modes of negative impulses input shaper, and then each different type single-mode input shapers are convolved and constitute the hybrid multi-mode negative impulses input shapers. They are built on the same principle, just change the order of convolution of the single-mode input shapers.

The single-mode NZV and NZVD input shapers are convolved which the first two natural frequencies and damping ratios are consistent, thereby form double-mode NZV-NZVD input shapers. For example, double-mode system, the first two modes natural frequencies are $76.6 \mathrm{~Hz}$ and $231.2 \mathrm{~Hz}$, damping ratio of the first two modes are 0.057 and 0.017 , respectively. The value $Q$ is set arbitrarily to be 1 . The parameters of each single-mode input shaper are:

$$
\begin{aligned}
& \mathrm{NZV} 1=\left[\begin{array}{l}
\mathrm{A}_{i} \\
t_{i}
\end{array}\right]=\left[\begin{array}{ccc}
1 & -2 & 2 \\
0 & 0.0029 & 0.00386
\end{array}\right] \\
& \mathrm{NZVD} 2=\left[\begin{array}{l}
\mathrm{A}_{i} \\
t_{i}
\end{array}\right]=\left[\begin{array}{ccccc}
1 & -2 & 2 & -2 & 2 \\
0 & 0.0006762 & 0.0012078 & 0.0027556 & 0.00295027
\end{array}\right]
\end{aligned}
$$


Convolving these two sets of impulses, the double-mode NZV-NZVD input shaper is:

$$
\mathrm{NZV}-\mathrm{NZVD}=\mathrm{NZV} 1 * \mathrm{NZVD} 2=\left[\begin{array}{l}
A_{i} \\
t_{i}
\end{array}\right]
$$

The multi-mode NZVD-NZV and NEI-NZV input shapers can improve the insensitivity and robustness of the shaper, too. The construction method is the same as the NZV-NZVD input shaper.

\section{Modeling of a 3-DOF Flexible Manipulator}

Fig. 3 shows that for a 3-DOF parallel manipulator which is composed of symmetrical arrangement of the three connecting rods for closed-loop type [10], each of connecting rod has a linear displacement constraint, and two rotational restraints. According to the LaGrange approach, the dynamic equation matrix of 3-DOF parallel manipulator is [4]:

$$
M \ddot{\eta}+K \eta=-M_{\rho} \ddot{\rho}-M_{\beta} \ddot{\beta}+F_{f g}
$$

In the equation: $M$ is the system modal mass matrix; $K$ is the modal stiffness matrix; $\eta$ is flexible generalized coordinates; $-M_{\rho} \ddot{\rho}-M_{\beta} \ddot{\beta}+F_{f g}$ is that the impact caused by the elastic vibration rigid body motion flexible connecting rod of due to elastic vibration; $F_{f g}$ is rigid in the elastic vibration caused by moving Modal force [10].

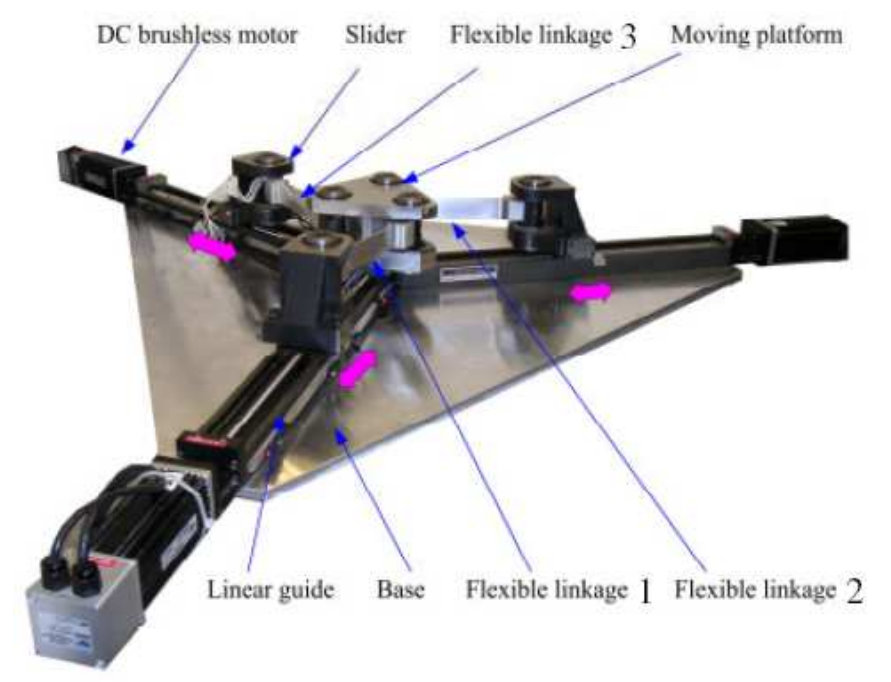

Fig. 3. Architecture of a3-DOF flexible parallel manipulator

Through numerical simulations, the first three modes frequencies are $70.5 \mathrm{~Hz}, 170.6 \mathrm{~Hz}$ and $280.8 \mathrm{~Hz}$ [10]. From the experimental model tests can obtain the first two modes frequencies and damping ratios which are $76.6 \mathrm{~Hz}, 231.2 \mathrm{~Hz}, 0.057$ and 0.017 , respectively.

\section{Digital Simulation}

The previous two natural frequencies and damping ratios of the 3-DOF manipulator's connecting rod can be obtained through experiments. And, $t_{1}=0$. The input signal of system is a unit step signal; it makes the sum of part continuous negative pulse amplitude of the multi-mode equal to 1 . Inhibit the residual vibration of system until it is less than $5 \%$. The each of NZV-NZVD, NZVD-NZV and NEI-NZV hybrid negative input shaper is composed of 15 pulses at least, the parameters of double-mode hybrid negative pulse NZV-NZVD, NZVD -NZV and NEI-NZV input shaper are in Tab. 1. 
The response curve of the 3-DOF system under the NZV-NZV input shaper and without input shaping is shown in Fig. 4. As illustrated in Fig. 4, the residual vibration is reduced effectively by double-mode negative NZV-NZV input shaping, and the delay time of the system response is shortened at the same time.

Table 1 The parameters of mixed double-mode negative input shapers

\begin{tabular}{cccccc}
\hline \multicolumn{2}{c}{ NZV-NZVD } & \multicolumn{2}{c}{ NZVD-NZV } & \multicolumn{2}{c}{ NEI-NZV } \\
\hline$A_{i}$ & $t_{i}(\mathrm{~s})$ & $A_{i}$ & $t_{i}(\mathrm{~s})$ & $A_{i}$ & $t_{i}(\mathrm{~s})$ \\
1 & 0 & 1 & 0 & 1 & 0 \\
-2 & 0.0006762 & -2 & 0.000932 & -2 & 0.000932 \\
2 & 0.0012078 & 2 & 0.001262 & 2 & 0.001262 \\
-2 & 0.0027556 & -2 & 0.00217 & -2 & 0.00224 \\
2 & 0.0029502 & 4 & 0.003102 & 4 & 0.00316 \\
-2 & 0.0029 & -4 & 0.003432 & -4 & 0.003502 \\
4 & 0.0035762 & 2 & 0.0037 & 2 & 0.00376 \\
-4 & 0.0041078 & -4 & 0.004632 & -4 & 0.004692 \\
4 & 0.0056556 & 4 & 0.004962 & 4 & 0.005022 \\
-4 & 0.0058502 & -2 & 0.00849 & -2 & 0.00853 \\
2 & 0.00386 & 4 & 0.009422 & 4 & 0.009462 \\
-4 & 0.0045362 & -4 & 0.009752 & -4 & 0.009792 \\
4 & 0.0050678 & 2 & 0.009 & 2 & 0.00907 \\
-4 & 0.0066156 & -4 & 0.009932 & -4 & 0.010002 \\
4 & 0.00681027 & 4 & 0.010262 & 4 & 0.010332 \\
\hline
\end{tabular}

The responses of different combinations of the hybrid double-mode negative impulses input shapers and the classic double-mode negative impulses input shapers are shown in Fig. 5-Fig. 7.

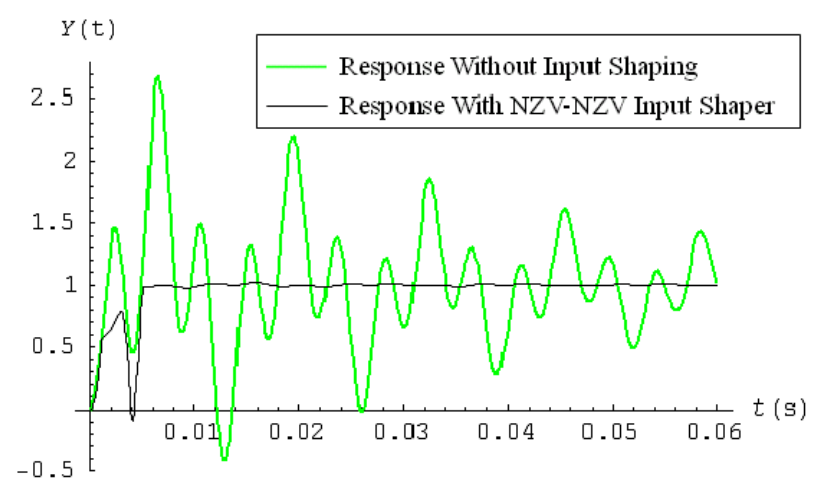

Fig. 4. Responses of NZV-NZV and without input shaping

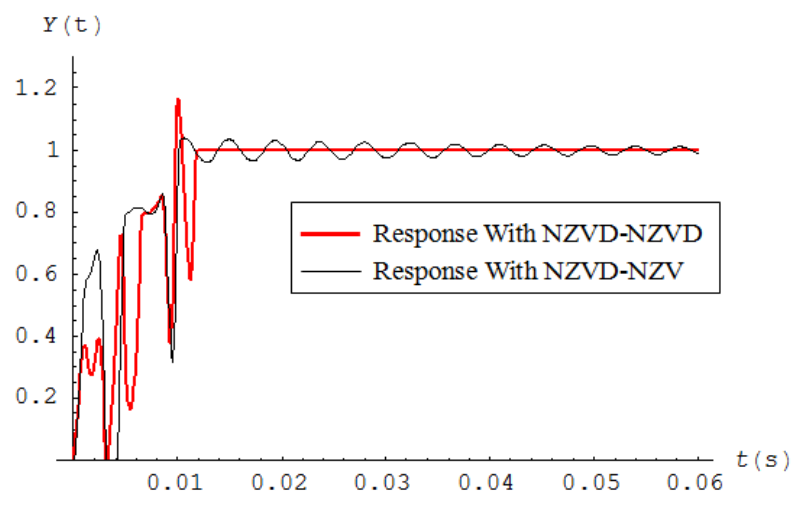

Fig. 6. Responses of NZVD-NZVD and NZVD-NZV

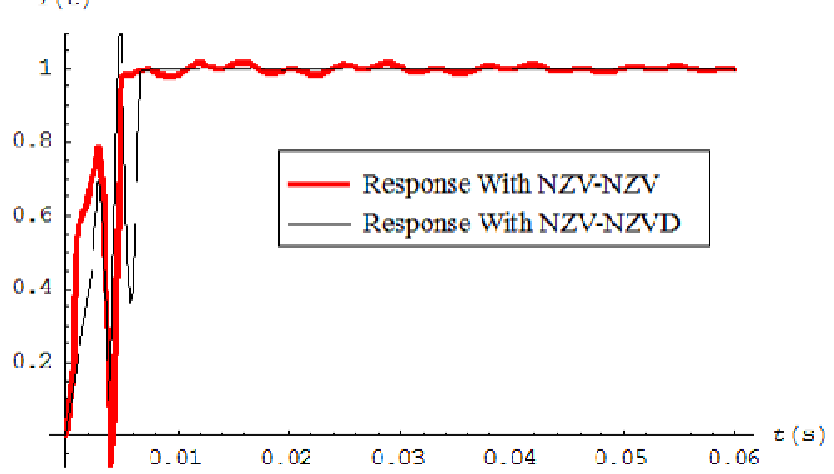

Fig. 5. Responses of NZV-NZV and NZV-NZV

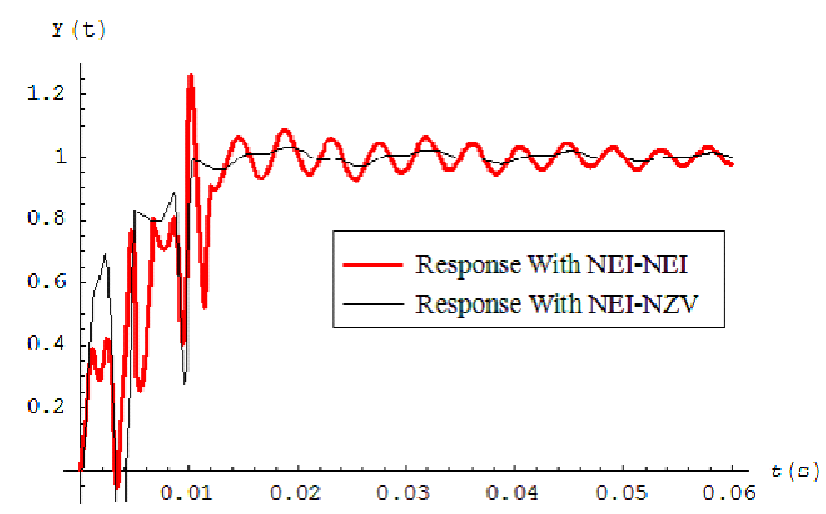

Fig. 7. Responses of NEI-NEI and NEI-NZV 
From Fig. 5-Fig. 7 can obtain the double-mode NZV-NZVD input shaper can suppress the residual vibration of the multi-mode system more effectively, but the response time delay. The double-mode NZVD-NZV and NEI-NZV input shapers have shortened the response time, however, the double-mode NZVD-NZV reduced the residual vibration of system more effectively than NEI-NZV input shapers. The output response time parameters of system from the different combinations of double-mode negative impulses input shapers are shown in Tab. 2.

Table 2 The parameters of system output response time

\begin{tabular}{ccccccc}
\hline Shapers & NZV-NZV & $\begin{array}{c}\text { NZV- } \\
\text { NZVD }\end{array}$ & $\begin{array}{c}\text { NZVD- } \\
\text { NZVD }\end{array}$ & $\begin{array}{c}\text { NZVD- } \\
\text { NZV }\end{array}$ & NEI-NEI & NEI-NZV \\
\hline $\begin{array}{c}\text { Response } \\
\text { time (s) }\end{array}$ & 0.0052 & 0.0064 & 0.012 & 0.01 & 0.0135 & 0.0102 \\
\hline
\end{tabular}

The sensitivity curve is an important parameter to evaluate the amplitude of residual vibration of a system. The width of the curve is the insensitivity of the shaper. It can more directly reflect the resonance frequency of system of the inhibitory effect of residual vibration. The sensitivity curves of different combinations input shaper are shown in Fig. 8 and Fig. 9.

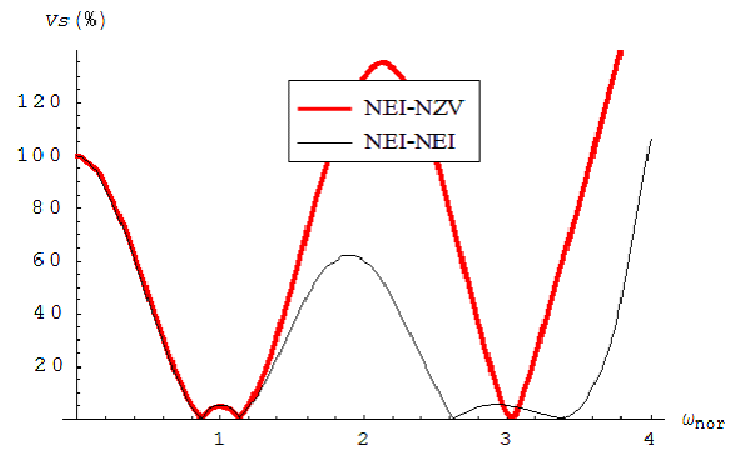

Fig. 8. Sensitivity curves of NEI-NEI and NEI-NZV

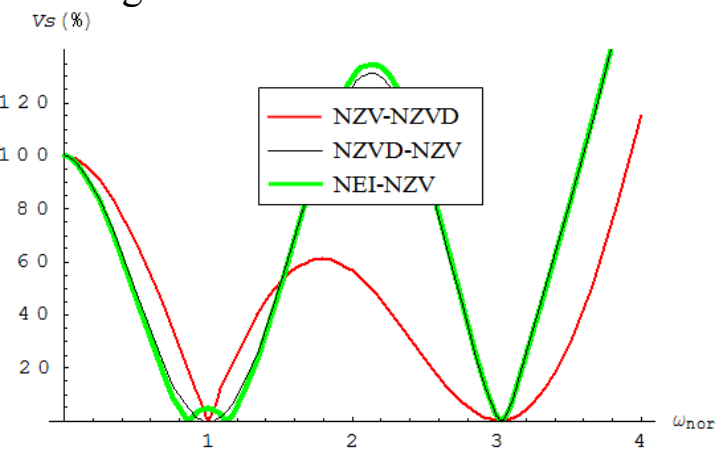

Fig. 9. Sensitivity curves of NZV-NZVD, NZVD-NZV and NEI-NZV

When the residual vibration is inhibited below $5 \%$, the insensitivity of different combinations of the hybrid multi-mode negative impulses input shapers is shown in Tab. 3.

Table 3 The insensitivity of NZV-NZVD, NZVD-NZV and NEI-NZV

\begin{tabular}{cccc}
\hline Input Shaper & NZV-NZVD & NZVD-NZV & NEI-NZV \\
\hline Insensitivity of first order & 0.1 & 0.3 & 0.4 \\
Insensitivity of second order & 0.41 & 0.14 & 0.14 \\
\hline
\end{tabular}

\section{Summary}

The principle and construction of the hybrid multi-mode NZV-NZVD, NZVD-NZV and NEI-NZV negative impulses input shaper are introduced. The classic double-mode NZV-NZV, NZVD-NZVD and NEI-NEI negative impulses input shapers and the hybrid double-mode negative impulses input shapers of a 3-DOF parallel manipulator are established, and compared the hybrid double-mode negative impulses input shapers with the classic double-mode negative impulses input shapers. Analyze the robustness of the shaper, the system response time delay and insensitivity with charts. According to numerical simulation results, both of the multi-mode input negative impulses shaper can be more effective in inhibiting the residual vibration of the system and shortening system response time delay. The robustness of the shapers is better, but the hybrid multi-modal negative pulse NZV-NZVD, NZVD-NZV and NEI-NZV input shaper's overall performance is better than anyone. 


\section{Acknowledgements}

This work was financially supported by the Zhejiang Qianjiang Talents Scheme Foundation (2010R10013).

\section{References}

[1] W. E. Singhose, W. P. Seering and N. C. Singer: Time-optimal negative input shapers, Journal of Dynamic, Measurement, and Control, 119(1997), p. 198.

[2] Youef-Toumia Kamal and Osamuito: A Time Delay Controller for Systems with Unknown Dynamics, ASME Journal of Dynamic Systems, 112(1990), p. 133.

[3] Z. Mohamed, A. K. Chee and A. W. I. Mohd-Hashim, et al: Techniques for vibration control of a flexible robot Manipulator, J. Robotica, 24(2006), p. 499.

[4] B. Li, X. P. Zhang and J. K. Mills: Vibration Suppression of a 3-PRR Flexible Parallel Manipulator Using Input Shaping, C. IEEE International Conference on Mechatronics and Automation, Changchun, China, 2009, p. 3539.

[5] M. D. Baumgart and L. Y. Pao: Discrete time-optimal command shaping, J. Automatica, 43(2007), p. 1403.

[6] F. D. Mohammed and N. M. Ziyad: Nonlinear Input - shaping Controller for Quay-side Container Cranes, J. Nonlinear Dynamics, 45(2006), p. 149.

[7] B. Li, L. Y. Xie and Y. L. Wei: Vibration Suppression Based on Negative Impuses Input Shaping for a 3-DOF Parallel Manipulator, Journal of Northeastern University (Natural Science), 10(2010), p. 1479.

[8] B. Li, L. Y. Xie and Y. L. Wei: Vibration Reduction of Flexible System by Time-Optimal Multi-mode Input Shaper, Journal of Northeastern University (Natural Science), 9(2010), p.1337.

[9] N. C. Singer, L. Y. Pao and W. E. Singhose: An Effcient Algo-rithm for the Generation of Multiple - Mode Input Shaping Sequences, IEEE International Conference on Control Applic-ations, Dearborn, MI, 1996, p. 373.

[10] X. P. Zhang, J. K Mills and W. L. Cleghorn: Dynamic modeling and experimental validation of a 3 PRR parallel manipulator with flexible intermediate links, Journal of Intelligent and Robotic Systems, 50(2007), p. 323. 